\title{
The effect of dissipation and an external magnetic field on the resonance dynamics of a domain wall in a five-layer ferromagnetic structure
}

\author{
A. M. Gumerov ${ }^{\dagger, 1}$, E. G. Ekomasov ${ }^{2,3,4}$, R. V. Kudryavtsev', M. I. Fakhretdinov ${ }^{1}$ \\ †article@solitonlab.com \\ ${ }^{1}$ Bashkir State University, 32 Zaki Validi St., Ufa, 450076, Russia \\ ${ }^{2}$ Tyumen State University, 6 Volodarskogo St., Tyumen, 625003, Russia \\ ${ }^{3}$ South Ural State University (National Research University), 76 Lenin Av., Chelyabinsk, 454080, Russia \\ ${ }^{4}$ Bashkir State Pedagogical University n. a. M. Akmulla, 3-a Oktyabrskoj revoljucii St., Ufa, 450008, Russia \\ ${ }^{5}$ Institute of Molecule and Crystal Physics UFRC RAS, 151 Oktyabrya Av., Ufa, 450075, Russia
}

\begin{abstract}
By the example of a five-layer ferromagnetic structure with two thin and three wide magnetic layers, the influence of attenuation and an external constant magnetic field on the dynamics of the domain wall (DW) is considered. It is shown that the features of the dynamics of the domain wall in a multilayer magnetic system in the presence of thin magnetic layers with a reduced value of the magnetic anisotropy constant are largely related to the resonant energy exchange between magnetic inhomogeneities. A diagram of the possible scenarios of the dynamics of the domain wall is constructed depending on the initial velocity of its movement and the distance between two identical thin magnetic layers. The presence of a critical distance was found that separates the dynamics of the domain wall into two regions with qualitatively different system behavior. In the first region, where the distance between the thin layers is rather small, the scattering of the domain wall occurs similarly to the case of the three-layer system studied earlier. In the second region, a much more complex dynamics of the scattering of the domain wall is observed, associated with the interaction of the domain wall and a localized nonlinear magnetization wave. The main effects of the collective influence of thin layers on the dynamics of the DW are preserved under the action of an external force, whereas when the DW moves by inertia, it almost completely disappears. A new dynamic effect of "quasitunneling" of the domain boundary, i.e. the passage of the domain boundary through the effective potential barrier, which appears due to the presence of two thin layers in the system at speeds lower than the minimum value for the case of a single thin layer. The result obtained - the existence of a range of parameters in which substantially less energy is required for a domain wall to pass through both thin layers can be interesting from a practical point of view.
\end{abstract}

Keywords: domain wall, localized magnetization wave, multilayer ferromagnet, resonant dynamics.

УДК: 537.611.3, 519.63

\section{Влияние затухания и внешнего магнитного поля}

\section{на резонансную динамику доменной границы в пятислойной ферромагнитной структуре}

\author{
Гумеров А. М. ${ }^{\dagger, 1}$, Екомасов Е. Г., ${ }^{2,3,}$, Кудрявцев Р. В. ${ }^{5}$, Фахретдинов М. И. ${ }^{1}$ \\ ${ }^{1}$ Башкирский государственный университет, ул. Заки Валиди, 32, Уфа, 450076, Россия \\ ${ }^{2}$ Тюменский государственный университет, ул. Володарского, 6, Тюмень, 625003, Россия \\ ${ }^{3} Ю$ жо-Уральский государственный университет (национальный исследовательский университет), \\ пр. Ленина, 76, Челябинск, 454080, Россия \\ ${ }^{4}$ Башкирский государственный педагогический университет им. М. Акмуллы, \\ ул. Октябрьской революции, 3-а, Уфа, 450008, Россия \\ ${ }^{5}$ Институт физики молекул и кристаллов УФИЦ РАН, пр. Октября, 151, Уфа, 450075, Россия
}


На примере пятислойной ферромагнитной структуры с двумя тонкими и тремя широкими магнитными слоями рассмотрено влияние затухания и внешнего постоянного магнитного поля на динамику доменной границы (ДГ). Показано, что особенности динамики доменной границы в мультислойной магнитной системе при наличии тонких магнитных слоев с уменьшенным значением константы магнитной анизотропии во многом связаны с резонансным обменом энергией между магнитными неоднородностями. Построена диаграмма возможных сценариев динамики доменной границы в зависимости от начальной скорости ее движения и расстояния между двумя одинаковыми тонкими магнитными слоями. Обнаружено наличие критического расстояния, разделяющего динамику доменной границы на две области с качественно различным поведением системы. В первой области, где расстояние между тонкими слоями достаточно мало, рассеяние доменной границы происходит аналогично случаю трехслойной системы, исследованному ранее. Во второй области наблюдается существенно более сложная динамика рассеяния доменной границы, связанная с взаимодействием доменной границы и локализованной нелинейной волны намагниченности. Основные эффекты коллективного влияния тонких слоев на динамику ДГ сохраняются при действии внешней силы и наличии затухания, тогда как при движении ДГ по инерции и при наличии затухания - практически полностью исчезают. Наблюдался новый динамический эффект «квазитуннелирования» доменной границы, т.е. прохождение доменной границы через эффективный потенциальный барьер, появляющийся из-за наличия двух тонких слоев в системе при скоростях, меньших минимального значения для случая одиночного тонкого слоя. Полученный результат - существование диапазона параметров, в котором для прохождения доменной границы через оба тонких слоя требуется существенно меньше энергии, может быть интересен с практической точки зрения.

Ключевые слова: доменная граница, локализованная волна намагниченности, мультислойный ферромагнетик, резонансная динамика.

\section{1. Введение}

Имеющие большие перспективы практического применения многослойные ферромагнитные структуры часто представляют собой периодически чередующиеся слои двух материалов с различными физическими свойствами [1]. Исследование даже одномерных моделей динамики спиновых волн и магнитных неоднородностей, распространяющихся в таких системах перпендикулярно границам раздела слоёв, приводит к новым интересным результатам [2-4]. Например, дает понимание влияния величин и знаков разных параметров на рассматриваемый процесс $[2,3,5,6]$. При исследовании динамики линейных и нелинейных волн намагниченности, распространяющихся перпендикулярно слоям, часто вводится пространственная модуляция магнитных параметров магнетика [2, 3, 6-8]. В таких системах возможна генерация локализованных волн намагниченности (ЛВН) типа магнитных солитонов и бризеров [7-10]. Наличие локализованных магнитных неоднородностей может существенно изменить динамику спиновых волн и доменных границ (ДГ) $[3,5,8,11]$. Особый интерес к магнитным солитонам и бризерам в настоящее время связан с появлением новых экспериментальных методик, позволяющих изучать процессы формирования и распространения локализованных волн намагниченности нанометровых размеров и взаимодействия их с доменными границами [12-16]. Проблему динамики ДГ в многослойных ферромагнетиках, представляющих собой структуры из чередующихся широких и тонких магнитных слоев с разными магнитными параметрами анизотропии, часто можно свести к задаче о взаимодействии кинков уравнения синус-Гордона УСГ с примесями. Она для одномерного случая обсуждается в литературе достаточно давно [17-21]. Важность учета возбуждения примесных мод в динамических задачах показана в [17-19]. Обна- ружен такой интересный эффект, как отражение кинка от притягивающей примеси из-за резонансного обмена энергией между кинком и примесной модой $[17,22]$.

Для более сложного и интересного случая пятислойной ферромагнитной структуры, с бесконечно тонкими слоями, без учета затухания и внешней силы в системе [23] найдено, что при малом расстоянии между тонкими слоями динамика ДГ аналогична динамике, наблюдаемой для случая трехслойной структуры. При больших расстояниях между тонкими слоями конечное состояние ДГ периодически меняется в зависимости от её начальной скорости. Для реальных материалов всегда характерно наличие диссипации, которая может принципиально влиять на динамическое поведение системы $[22,24,25]$. Влияние затухания и внешнего магнитного поля на возникновение резонансных эффектов для случая трехслойной структуры было исследовано в [22]. Показано, что затухание и внешняя сила противодействуют возникновению резонансного отражения ДГ от притягивающего тонкого слоя. Однако, вызывающая его причина - резонансный обмен энергией между магнитными неоднородностями - по-прежнему имеет место. В настоящей работе исследуется влияние внешнего магнитного поля и диссипации на резонансную динамику доменных границ в пятислойной ферромагнитной структуре с учётом возможности возбуждения ЛВН.

\section{2. Динамика ДГ в пятислойной ферромагнитной структуре}

Рассмотрим пятислойную ферромагнитную структуру, состоящую из трех широких слоев, разделенных двумя тонкими слоями, находящимися на расстоянии $d$ друг от друга. Широкие и тонкие магнитные слои отличаются друг от друга значениями константы магнитной анизотропии [26]. Ширину ДГ и тонкого магнитного слоя $W$ считаем величинами одного и того же порядка. 
Пространственную модуляцию константы магнитной анизотропии в безразмерной форме будем моделировать в виде [27]:

$$
K(x)=\left\{\begin{array}{l}
1, \quad x<x_{1}, x_{1}+W<x<x_{1}+W+d, x>x_{1}+2 W+d, \\
1-\Delta K, x_{1} \leq x \leq x_{1}+W, x_{1}+W+d \leq x \leq x_{1}+2 W+d,
\end{array}\right.
$$

где $K(x)=K_{1}(x) / K_{1}^{0}, K_{1}^{0}-$ константа анизотропии в толстых слоях. Очевидно, что при $\Delta K>0$ тонкий слой является потенциальной ямой для ДГ, а при $\Delta K<0-$ потенциальным барьером. При решении динамических задач удобно перейти к сферическим координатам вектора намагниченности $M(\sin (\varphi), \cos (\varphi) \sin (\theta), \cos (\varphi)$ $\cos (\theta))$, где $0 \leq \theta \leq 2 \pi$ - угол в плоскости $y z$ между направлением вектора магнитного момента и осью лёгкого намагничивания (ось $O z),-\pi / 2<\varphi<\pi / 2-$ угол, описывающий выход $M$ из плоскости доменной границы. Учитывая в плотности энергии магнетика обменное взаимодействие и анизотропию и считая $\varphi \ll 1$, уравнение движения для намагниченности в угловых переменных можно представить в следующем обезразмеренном виде [8,28]:

$$
\Delta \theta-\ddot{\theta}-\frac{1}{2} K(x) \sin (2 \theta)=h \sin (\theta)+\alpha \dot{\theta},
$$

где $h=\left(H_{z} / 4 \pi M_{S}\right) Q^{-1}-$ нормированное внешнее магнитное поле, $\alpha=\alpha_{0} / \sqrt{Q}-$ нормированная константа затухания, здесь $Q=K_{1} /\left(2 \pi M_{S}^{2}\right)$ - фактор качества материала, $a_{0}-$ константа затухания. Время $t$ нормировано на $4 \pi M_{s} \gamma \sqrt{Q}$, координата $x$ нормирована на $\delta_{0}$, где $\delta_{0}-$ ширина статической блоховской ДГ. При получении уравнения (2) считалось, что, $K_{1} \ll 2 \pi M_{S}^{2}$. Уравнение (2) при нулевой правой части и $K(x)=1$ переходит в известное уравнение синус-Гордона [17].

Уравнение (2) решалось численно, с использованием явной схемы [22]. Дискретизация уравнения проводилась по стандартной пятиточечной схеме типа «крест», обладающей условием устойчивости $(\Delta t / \Delta x)^{2} \leq 0.5$, где $\Delta t-$ шаг по времени, $\Delta x-$ шаг по координате. В начальный момент времени имеем ДГ блоховского типа, движущуюся с постоянной скоростью $v_{0}$, а граничные условия имеют вид: $\theta(-\infty, t)=0, \theta(+\infty, t)=\pi, \theta^{\prime}( \pm \infty, t)=0$.

Отметим, что программы для численного решения уравнения (2), которые использовались ранее в работах $[7,22,29]$, позволяют рассчитать структуру и динамику ДГ с точностью достаточной, чтобы наблюдать захват (или «пиннинг») и прохождение доменной границы через мультислойную структуру и свойства возбуждаемых нелинейных волн. Однако, для изучения возможных резонансных эффектов необходима принципиально более высокая точность счета. Поэтому при аппроксимации функции $\theta(x, t)$ использовалось $N_{x}=10^{4}$ точек. В связи сособыми требованиями кточности проводимых расчетов, реализован и особый контроль погрешности результата. Кроме того, используемая схема удобна тем, что является «одношаговой», использует сравнительно небольшое количество обращений к памяти и обладает потенциалом для оптимизации вычислительного алгоритма. Также, она с минимальными изменениями может быть адаптирована и для многомерных вариантов уравнения движения (2).
В ходе численного эксперимента ДГ пересекает области тонких слоев, при этом в каждый момент времени рассчитывается структура ДГ и ее основные динамические характеристики: положение центра, скорость и траектория движения. Вначале рассмотрим простой случай движения по инерции и отсутствия внешней силы. На Рис. 1 представлена диаграмма возможной эволюции динамики доменной границы в зависимости от начальной скорости ДГ $v_{0}$ и расстояния между тонкими слоями $d$. Из Рис. 1 видно наличие некоторого критического расстояния между тонкими слоями $d_{\text {crit }}$, на котором качественно меняется динамика ДГ. В приведенном случае это значение $d_{\text {crit }} \approx 3.2$. При $d<d_{\text {crit }}$ два тонких слоя можно в целом рассматривать как эффективный одиночный слой. На Рис. 1 горизонтальная линия соответствует пороговой скорости прохождения одиночного тонкого слоя $\cup_{\min }^{\text {one }}$. В одной точке $(d=0.84)$ совпадают пороговые скорости, необходимые для прохождения через тонкий слой как для случая двух, так и одинокого тонкого слоя с теми же параметрами. Обнаружены следующие сценарии динамики ДГ: «захватывается» в области первого тонкого слоя (область 2), или второго тонкого слоя (область 3), ДГ осциллирует между ними продолжительное время (область 1), отражается в обратном направлении (Рис. 1, область 5), либо проходит область тонких слоев (Рис. 1, область 4). В последних двух случаях в области тонких слоев возбуждаются осциллирующие локализованные высокоамплитудные нелинейные волны намагничен-

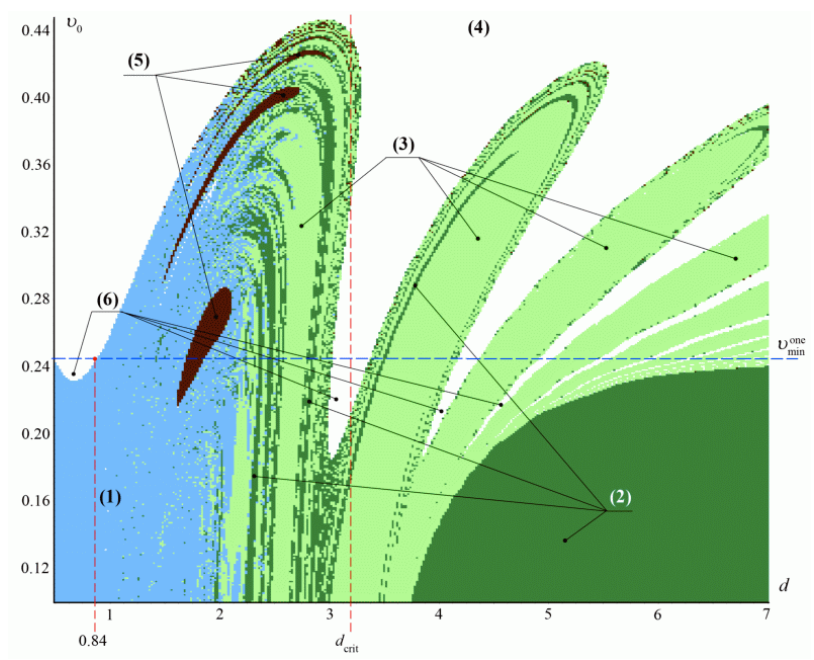

Pис. 1. (Color online) Диаграмма возможных сценариев динамики движущейся по инерции ДГ в зависимости от ее начальной скорости $v_{0}$ и расстояния $d$ между тонкими слоями при $W=1$, $\Delta K=0.8$. Области диаграммы: (1) - захват ДГ на одном из тонких слоев с длительным перескоком, (2) - захват на первом тонком слое, (3) - захват на втором тонком слое, (4) - прохождение ДГ через оба тонких слоя, (5) - полное отражение ДГ, (6) - область «квазитуннелирования», является частью области (4).

Fig. 1. (Color online) Diagram of possible scenarios of the dynamics of a DW moving by inertia depending on its initial velocity $v_{0}$ and the distance $d$ between thin layers at $W=1, \Delta K=0.8$. Areas of the diagram: (1) - capture of the DW on one of the thin layers with a long hop, (2) - capture on the first thin layer, (3) - capture on the second thin layer, (4) - passage of the DW through both thin layers, (5) - full reflection of the DW, (6) - the region of "quasitunneling", is part of the region (4). 
ности бризерного типа, которые существенно влияют на динамику ДГ. Во-первых, на их возбуждение может тратиться значительная часть начальной энергии ДГ. Во-вторых, последующее взаимодействие с этими локализованными волнами лежит в основе механизма резонансных эффектов. Также можно выделить случай, когда тонкие слои расположены достаточно близко друг к другу, тогда энергия, необходимая для перехода между ними, невелика и ДГ может осциллировать между ними продолжительное время. Отметим, что подобное динамическое поведение ДГ было получено нами ранее и для случая бесконечно тонких слоев [23], когда вместо функции (1) использовалась дельта-функция. Можно ожидать, что и изменение функции (1) на функцию гиперболического вида, как для случая трехслойной структуры [8], не приведет к качественному изменению динамического поведения ДГ.

Исследуем далее все возможные варианты инерционной динамики ДГ при наличии затухания в системе в зависимости от величины $v_{0}$ и $d$. На Рис. 2 представлена диаграмма возможных вариантов динамики ДГ, движущейся по инерции. Видно, что по сравнению с бездиссипативным случаем (см. Рис. 1), диаграмма качественно изменилась. В частности, исчез ее «лепестковый» характер, при котором области захвата ДГ и ее прохождения чередовались при одном и том же значении параметра $d$. Вместо этого появились сплошные области захвата на первом (область 1) и втором (область 2) тонких слоях, которые значительно расширились. Областей квазитуннелирования (см. Рис. 1, область 6), при которых наблюдается прохождение ДГ через оба тонких слоя при скорости, меньшей чем пороговая скорость $\mathrm{v}_{\min }^{\text {one }}$ прохождения через одиночный тонкий слой с константой анизотропии вида $K(x)=1-\Delta K$, где $\Delta K \neq 0$ при $x_{0} \leq x \leq x_{0}+W$ с теми же значениями $W$ и $\Delta K$, также не удалось обнаружить. Однако между рассматриваемыми диаграммами есть и несколько схожих черт. Например, при малых значениях параметра $d<1.5$ наблюдается чередование областей захвата на первом и втором тонком слое. Аналогичная область в бездиссипативном случае выделена на Рис. 1 как область захвата с длительным перескоком. Кроме этого, существует критическое расстояние между тонкими слоями $d_{\text {crit }} \approx 4$ вблизи минимума пороговой скорости прохождения через оба тонких слоя $v_{\min }^{\mathrm{II} \rightarrow \mathrm{III}}(d)$ (то есть граница между областями 2 и 3). Однако величина $d_{\text {crit }}$ уже не несет того смысла, которым она обладает в бездиссипативном случае (как граница, разделяющая две области качественно различного динамического поведения системы), поскольку теперь при каждом значении $d$ существует единственное значение $v_{\min }^{\text {II } \rightarrow \text { II }}(d)$. Значение $d_{\text {crit }}$ заметно отличается от случая представленного на Рис. 1. Также необходимо отметить тот факт, что аналогично бездиссипативному случаю наличие второго тонкого слоя «способствует» прохождению ДГ через первый тонкий слой и снижает минимально необходимую для этого скорость, поэтому при $d \rightarrow 7$ граница перехода из области 1 в область $2\left(v_{\min }^{\mathrm{I} \rightarrow \mathrm{II}}(d=7)=0.513\right)$ лежит заметно ниже пороговой скорости прохождения через одиночный тонкий слой $\mathrm{U}_{\min }^{\text {one }} \approx 0.53$.
Таким образом, появление затухания привело к существенному упрощению структуры диаграммы возможной динамики ДГ. Из диаграммы исчезли резонансные окна прохождения через область, содержащую два тонких слоя, поскольку данный эффект был обусловлен тем, что малоамплитудные спиновые волны, излучаемые при пересечении ДГ первого тонкого слоя, возбуждали ЛВН на втором тонком слое. Учет диссипации приводит как к заметному затуханию излучаемых волн, так и к ослаблению влияния ЛВН. Однако ранее в [22] для случая одного тонкого слоя было показано, что затухание не приводит к принципиальной невозможности резонансного обмена энергией между ДГ и ЛВН, поэтому при достаточно малых значениях параметра а все же не исключено наличие резонансных окон прохождения ДГ через область, содержащую оба тонких слоя.

Рассмотрим далее случай, когда ДГ при учете затухания движется под воздействием внешнего магнитного поля. Диаграмма возможных сценариев динамики ДГ приведена на Рис. 3. Структура диаграммы похожа на структуру диаграммы в бездиссипативном случае на Рис. 1. Это проявляется в первую очередь в том, что существует критическое значение расстояния между тонкими слоями $d_{\text {crit }} \approx 3.1$, которое разделяет рисунок на две качественно различные области. При $d>d_{\text {crit }}$ диаграмма приобретает лепестковый характер. Причем значение $d_{\text {crit }}$ незначительно отличается от случая на Рис. 1. Однако между ними есть и ряд существенных отличий. Как и в предыдущем рассмотренном случае (Рис. 2) отсутствует область квазитуннелирования (см. Рис. 1, область 6). Структура самих областей значительно

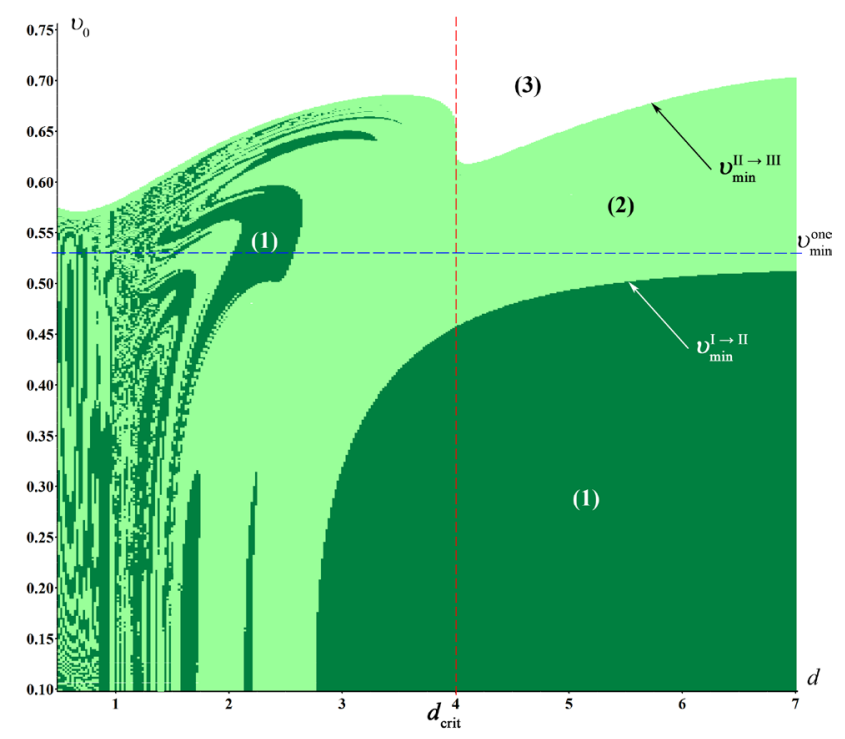

Pис. 2. (Color online) Диаграмма возможных сценариев динамики движущейся по инерции ДГ в зависимости от параметров $v_{0}$ и $d$ при $W=1, \Delta K=0.8$ и $\alpha=0.02$. Области диаграммы: (1) - захват ДГ на первом тонком слое, (2) - захват ДГ на втором тонком слое, (3) - прохождение ДГ через область обоих тонких слоев.

Fig. 2. (Color online) Diagram of possible scenarios of dynamics moving along the inertia of a DW, depending on the parameters $v_{0}$ and $d$ at $W=1, \Delta K=0.8$, and $a=0.02$. Areas of the diagram: (1) - DG capture on the first thin layer, (2) - DG capture on the second thin layer, (3) - DG passage through the region of both thin layers. 


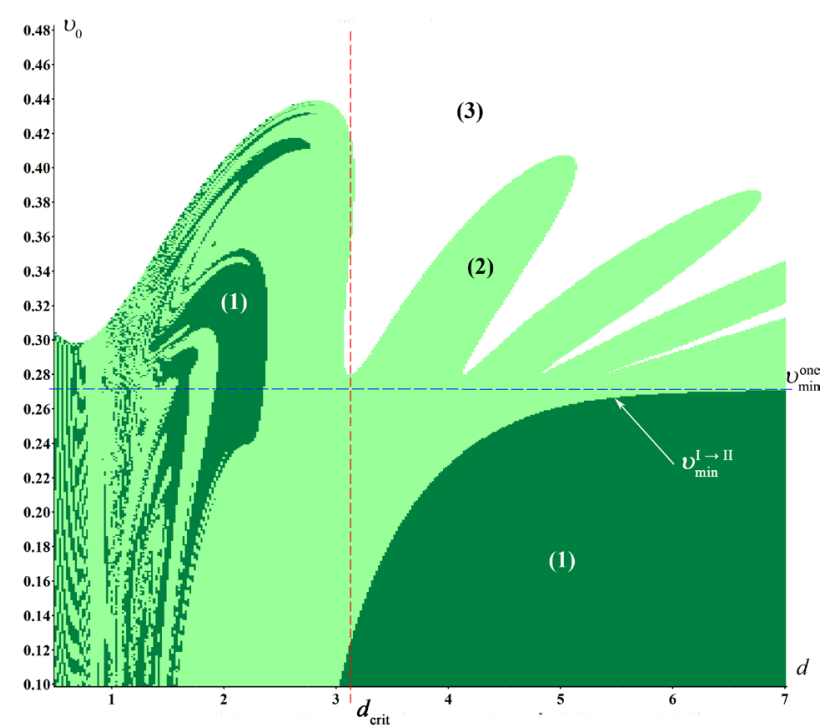

Pис. 3. (Color online) Диаграмма возможных сценариев динамики ДГ, движущейся под действием внешнего магнитного поля, в зависимости от параметров $v_{0}$ и $d$ при $W=1, \Delta K=0.8$ и $\alpha=0.02$. Области диаграммы: (1) - захват ДГ на первом тонком слое, (2) - захват ДГ на втором тонком слое, (3) - прохождение ДГ через область обоих тонких слоев.

Fig. 3. (Color online) Diagram of possible scenarios of the dynamics of a DW moving under the action of an external magnetic field, depending on the parameters $v_{0}$ and $d$ at $W=1, \Delta K=0.8$, and $\alpha=0.02$. Areas of the diagram: (1) $-D G$ capture by the first thin layer, (2) - DG capture on the second thin layer, (3) - DG passage through the region of both thin layers.

упростилась, и они стали более однородными. Например, при $d>d_{\text {crit }}$ и $v_{0}>v_{\min }^{\text {one }}$ исчезли узкие полосы области 1 , поскольку действие внешней силы $h>0$ увеличивает вероятность локализации ДГ на втором тонком слое. По этой же причине пороговая скорость прохождения через первый тонкий слой $v_{\min }^{\mathrm{I} \rightarrow \mathrm{I}}$ (то есть граница раздела между областями 1 и 2) достаточно быстро асимптотически приближается к пороговой скорости для одного тонкого слоя $\lim _{d \rightarrow 7} v_{\min }^{\mathrm{I} \rightarrow \mathrm{II}}(d) \rightarrow \mathrm{v}_{\text {min }}^{\text {one }} \approx 0.271$.

\section{3. Заключение}

Рассмотрено влияние затухания и внешнего постоянного магнитного поля на динамику ДГ в пятислойной ферромагнитной структуре. Показано, что коллективные эффекты влияния наличия тонких слоев (с уменьшенным значением константы магнитной анизотропии) в системе на динамику ДГ во многом связаны с резонансным обменом энергией между магнитными неоднородностями. Построена диаграмма возможных сценариев динамики ДГ в зависимости от ее начальной скорости и расстояния между двумя тонкими слоями. Обнаружено наличие критического расстояния между ними, разделяющего зависимость на две области с качественно различным динамическим поведением системы. В первой области, где расстояние между слоями достаточно мало, динамика ДГ происходит аналогично случаю одиночного тонкого слоя. Во второй - наблюдается существенно более сложная динамика ДГ, связанная с взаимодействием ДГ и локализованной в области тонкого слоя нелинейной волны намагниченности. Основные эффекты коллективного влияния тонких слоев на динамику ДГ сохраняются при действии внешней силы и наличии затухания, тогда как при движении ДГ при наличии затухания по инерции - практически полностью исчезают.

Благодарности/Acknowledgements. Статья выполнена при поддержке Правительства РФ (Постановление No 211 om 16.03.2013 2.), согташение No 02.A03.21.0011. Для А. М. Гумерова и Р. В. Кудрявиева работа поддержана грантом РФФИ, проект 18-31-00122. / The work was supported by Act 211 Government of the Russian Federation, contract No 02.A03.21.0011. For A.M. Gumerov and R. V. Kudryavtsev's work was supported by a grant from the Russian Foundation for Basic Research, project 18-31-00122.

\section{Литература/References}

1. D.D. Tang, Yu.-J.Le. Magnetic Memory Fundamentals and Technolog. Cambridge, Cambridge University Press, New York (2010) 196 p.

2. V.V. Kruglyak, C.S. Davies, V.S. Tkachenko, O.Y. Gorobets, Y.I. Gorobets, A.N. Kuchko. Journal of Physics D: Applied Physics. 50 (9), 094003 (2017). Crossref

3. E. Della Torre, C. M. Perlov. J. Appl. Phys. 69 (8), 4596 (1991). Crossref

4. Y. Gusieva, P. Graczyk, O. Gorobets, M. Krawczyk. Acta Physica Polonica A. 133 (3), 489 (2018). Crossref

5. E.G. Ekomasov, Sh.A. Azamatov, R.R. Murtazin. Phys. Met. Metallogr. 105 (4), 313 (2008). Crossref

6. M.A. Shamsutdinov, V.N. Nazarov, I.U. Lomakina, A.T. Kharisov, D.M. Shamsutdinov. Ferro- i antiferromagnitodinamika. Nelineynyye kolebaniya, volny i solitony. Moscow, Nauka (2009) 456 p. (in Russian) [М.А. Шамсутдинов, И.Ю. Ломакина, В.Н. Назаров, А.Т. Харисов, Д. М. Шамсутдинов. Ферро- и антиферромагнитодинамика. Нелинейные колебания, волны и солитоны. Москва, Наука (2009) 456 с.]

7. E.G. Ekomasov, A.M. Gumerov, R.R. Murtazin, R.V. Kudryavtsev, A.E. Ekomasov, N.N. Abakumova. Solid State Phenomena. 233 - 234, 51 (2015). Crossref

8. E. G. Ekomasov, R. R. Murtazin, V.N. Nazarov. Journal of Magnetism and Magnetic Materials. 385, 217 (2015). $\underline{\text { Crossref }}$

9. A.M. Gumerov, E.G. Ekomasov, R.V. Kudryavtsev, M. I. Fakhretdinov. Letters on Materials. 8 (3), 299 (2018). Crossref

10. E. G. Ekomasov, R.R. Murtazin, O.B. Bogomazova, A. M. Gumerov. JMMM. 339, 133 (2013). Crossref

11. V. V. Kiselev, A. A. Rascovalov. Chaos, Solitons \& Fractals. 45, 1551 (2012). Crossref

12. K.S. Novoselov, A.K. Geim, S. V. Dubonos, E.W. Hill, I. V. Grigorieva. Nature. 426, 812 (2003). Crossref

13. R. Kukreja, S. Bonetti, Z. Chen, D. Backes, Y. Acremann, J.A. Katine, A. D. Kent, H.A. Dürr, H. Ohldag, J. Stöhr. Phys. Rev. Lett. PRL. 115, 096601 (2015). Crossref

14. J. P. Tetienne, T. Hingant, J. V. Kim, L. H. Diez, J. P. Adam, K. Garcia, J. F. Roch, S. Rohart, A. Thiaville, D. Ravelosona, V. Jacques. Science. 344, 1366 (2014). Crossref 
15. M.V. Gerasimov, M.V. Logunov, A.V. Spirin, Yu. N. Nozdrin, I. D. Tokman. Phys. Rev. B. 94, 014434 (2016). Crossref

16. D. Backes, F. Macià, S. Bonetti, R. Kukreja, H. Ohldag, A. D. Kent. PRL. 115 (12), 127205 (2015). Crossref

17. The Sine-Gordon Model and Its Applications: From Pendula and Josephson Junctions to Gravity and High-energy Physics. V.10. (Ed. by J. Cuevas-Maraver, P.G. Kevrekidis, F. Williams). Springer (2014) 263 p. Crossref

18. T. Dauxois, M. Peyrard. Physics of Solitons. New York, Cambridge University Press (2010).

19. O. M. Brown, J. S. Kivshar. The Frenkel-Kontorova model: Concepts, methods, and applications. Springer (2004) $519 \mathrm{p}$.

20. M. B. Fogel, S. E. Trullinger, A. R. Bishop, J. A. Krumhandl. Phys. Rev. B. 15, 1578 (1977). Crossref

21. O. M. L. Gomide, M. Guardia, T.M. Seara. Journal of Differential Equations. 269(4), 3282 (2020). Crossref

22. E. G. Ekomasov, A. M. Gumerov, R. V. Kudryavtsev. JETP Letters. 101, 835 (2015). Crossref
23. E. G. Ekomasov, A. M. Gumerov. Letters on materials. 3 (2), 103 (2013). (in Russian) [A. М. Гумеров, Е. Г. Екомасов. Письма о материалах. 3 (2), 103 (2013).] Crossref

24. D. Saadatmand, S. V.Dmitriev, D. I. Borisov, P. G. Kevrekidis. Phys. Rev. E. 90 (5), 052902 (2014). Crossref

25. E. G. Ekomasov, R.V. Kudryavtsev, A.M. Gumerov. Letters on Materials. 7 (2), 160 (2017). (in Russian) [Е. Г. Екомасов, Р. В. Кудрявцев, А. М. Гумеров. Письма о материалах. 7 (2), 160 (2017).] Crossref

26. E.G. Ekomasov, A.M. Gumerov, R.V. Kudryavtsev, S. V. Dmitriev, V. N. Nazarov. Brazilian Journal of Physics. 48 (6), 576 (2018). Crossref

27. E. G. Ekomasov, A.M. Gumerov. Letters on materials. 4 (4), 237 (2014). $\underline{\text { Crossref }}$

28. E. G. Ekomasov, A.M. Gumerov, R. V. Kudryavtsev. Letters on materials. 6 (2), 138 (2016). (in Russian) [Е. Г. Екомасов, А. М. Гумеров, Р. В. Кудрявцев. Письма о материалах. 6 (2), 138 (2016).] Crossref

29. E. G. Ekomasov, A.M. Gumerov, R.R. Murtazin. Mathematical Methods in the Applied Sciences. 40 (17), 6178 (2016). $\underline{\text { Crossref }}$ 